\title{
Agilité et conception de filière : le cas de la montre
} SWATCH

\section{Agility and Product Supply Chain Design: the Case of the Swatch}

\author{
Brunelle Marche ${ }^{1}$, Vincent Boly ${ }^{1}$, Laure Morel ${ }^{1}$, Frédérique Mayer ${ }^{1}$, Roland Ortt $^{2}$ \\ ${ }^{1}$ ERPI, Université de Lorraine, Nancy, France, brunelle.marche@univ-lorraine.fr, vincent.boly@univ-lorraine.fr, \\ laure.morel@univ-lorraine.fr, frederique.mayer@univ-lorraine.fr. \\ ${ }^{2}$ Faculty of Technology, Policy and Management, Delft, the Netherlands, j.r.ortt@tudelft.nl
}

RÉSUMÉ. Lors du lancement de nouveaux produits, les entreprises innovantes doivent anticiper l'organisation de la filière, supportant la fabrication du produit et que de fait, elles doivent aussi redéfinir leur rôle au sein de cette dernière pour rester compétitive. Une réflexion doit donc être menée dès la conception du nouveau produit afin d'anticiper les changements organisationnels, technologiques ou stratégiques occasionnés. Une filière « dite » agile facilite l'intégration d'un nouveau produit en limitant les risques induits par ces différents changements. Dans cet article, nous montrerons qu'anticiper la conception de la filière, au plus tôt dans le processus de développement du nouveau produit, permet de mettre en œuvre les stratégies les plus efficaces pour la rendre agile et donc plus apte à supporter les aléas et les incertitudes découlant de l'innovation. Pour illustrer nos propos, le cas emblématique de la Swatch est étudié.

ABSTRACT. During the launch of new products, innovative companies must be ready for a reorganization of the supply chain that supports the manufacturing of the product in question. Thus, they have to redefine their role at the heart of manufacturing in order to remain competitive. Therefore, reflection must take place as soon as the new product is designed in order to anticipate the organizational, technological and strategic changes. An "agile" supply chain facilitates the integration of a new product by limiting the risks induced by these different changes. In this article, we will show that anticipating the design of the supply chain as early as possible in the process of development of the new product allows us to implement the most effective strategies to make it agile, and therefore more able to withstand the risks and uncertainties arising from innovation. To illustrate our points, the emblematic case of the Swatch is studied.

MOTS-CLÉS. Filière, Agilité, Agile, Modélisation.

KEYWORDS. Supply chain, Agility, Agile, Modelling.

\section{Introduction}

L'émergence d'un produit innovant transforme l'organisation interne d'une entreprise [GAR 02] ainsi que son écosystème [OCO 13], et plus particulièrement la séquence d'entreprises appelée filière qui supporte sa fabrication. En effet, à chaque produit correspond une séquence d'entreprises impliquées dans des activités de transformation et de distribution - depuis l'approvisionnement de la matière première, la fabrication du produit jusqu'à la distribution à un client final - [CHR 92; BID 13]. La filière se construit progressivement afin d'optimiser sa séquence d'activités.

De fait, une filière est spécifique a un produit et donc n'est pas nécessairement adaptée au produit innovant en émergence. Une réflexion sur la future filière devient essentielle pour les acteurs impliqués dans l'innovation. Ainsi, co-concevoir le futur produit et la filière associée, au plus tôt, participe au succès des innovations sur le marché. Cette conception intégrée de la filière dans les phases amont de l'innovation suppose une anticipation des modifications organisationnelles sur cette dernière ou une adaptation de celle-ci pour répondre de manière optimale aux spécifications du futur produit [VON 06]. Nous parlerons alors de filière agile. Une filière agile est un ensemble d'acteurs réactif, dynamique et capable de s'adapter aux changements de son environnement.

Dans nos travaux, nous cherchons à confronter le concept d'agilité avec le corpus de connaissance relatif à la mise en place d'une filière afin de confirmer qu'anticiper la conception de filière au plus tôt dans le processus de développement du nouveau produit rend plus agile et donc plus innovante une 
organisation. Pour ce faire, nous avons choisi d'illustrer notre recherche à l'aide d'un cas emblématique, la Swatch. Notre objectif est de détailler les stratégies mises en œuvre par l'entreprise pour configurer de manière optimale la filière du produit innovant. Cette étude vise à montrer que la filière initiale a été rendue agile pour favoriser la réussite du nouveau produit sur le marché dès son lancement.

Dans une première partie, une revue de l'état de l'art nous permettra de présenter les concepts clés de notre recherche à savoir le produit et le système produit, la filière et le système filière puis la notion d'agilité. Plus particulièrement, la dimension «systèmes inter-reliés » du produit et de la filière sera présentée ainsi que la notion de filière agile et d'agilité de filière. Une seconde partie présente la méthodologie de recherche. La troisième partie présente les résultats de notre étude de cas pour être ensuite discutés dans un quatrième paragraphe. Enfin, la conclusion de la recherche est présentée.

\section{1. État de l'art}

\subsection{Définition du produit et de la filière dans une perspective systémique}

\subsubsection{Définition du produit et d'une filière}

Un produit est défini par des composants liés entre eux par le biais d'un jeu d'interfaces, constituant ainsi l'architecture de ce produit [HEN 90]. Son architecture représente sa structure fondamentale, elle décrit de manière exhaustive l'ensemble des caractéristiques incluant le nombre et le type de composants et d'interfaces entre ces composants [ULR 95 ; FIX 05].

La fabrication d'un produit mobilise un ensemble d'entreprises, constituant une filière. Une filière est un réseau d'entreprises impliquées dans des processus, en amont et en aval, qui crée de la valeur sous la forme d'un produit pour un client final [CHR 92]. Elle est caractérisée par un ensemble d'opérations ou procédés [BID 13], par une succession d'activités à valeur ajoutée [GER 05] nécessaires pour passer d'une matière première à un produit final.

Une filière est donc composée d'entreprises (fournisseurs, fabricants, distributeurs...) reliées entre elles par des flux physiques, mais aussi financiers et informationnels [LAP 00] et ils sont directement liés au produit [LAB 85].

Une filière est donc un ensemble regroupant des relations et des flux entre un ensemble d'opérations et de processus qui génèrent de la valeur sous la forme d'un produit pour un consommateur final [SLA $16]$.

L'une des variables constitutives d'une filière est représentée par les processus de chacune des entités qui la composent. Un processus est un agencement ordonné d'activités [BON 06]. Une activité représente le «travail quotidien » des employés d'une entreprise et fait appel à leur savoir-faire [LOR 03]. La finalité d'un processus porte donc sur la transformation progressive des entrées en résultats attendus, représentant ainsi une chaîne de valeur ajoutée [BON 06]. Le processus est donc associé à des actions et des intentions des individus. On peut faire l'hypothèse que les changements (en particulier dus à une innovation dans notre cas), sur le processus de chaque entité de la filière auront des conséquences importantes sur les hommes (actions et intentions) et sur la valeur générée (intention). De ce fait, l'étude de la filière d'un point de vue processus est privilégiée ici.

\subsubsection{Le produit et la filière d'un point de vue systémique}

Un système peut être vu comme une unité globale dynamique organisée où les éléments, actions et individus qui la composent sont inter-reliés et organisés selon un but [MOR 77 ; DER 75]. Ainsi, un système est le résultat de l'agencement de relations entre des composants ou individus, où la représentation de cet agencement peut être considérée comme l'architecture du système [BON 06]. 
L'étude d'une architecture aborde les aspects structurels (les éléments et leurs relations) et temporels (la dynamique de fonctionnement) [MEI 02].

De ce fait, l'approche systémique facilite l'étude de systèmes dans leur complexité. En se basant sur cette approche, un produit ainsi que sa filière peuvent être considérés comme des systèmes inter-reliés.

L'architecture du produit exige l'implication de plusieurs entreprises : chaque composant, chaque interface réclame un savoir-faire souvent spécifique. Ainsi, l'ensemble architecturé des composants mobilise des procédés et des compétences distribués entre un ensemble d'acteurs, à la fois capable de maitriser une phase mais aussi d'agir collectivement avec les autres. De ce fait, l'architecture du produit influence l'architecture de la filière. Ces architectures sont évolutives, par des décisions de conception sur le produit ou par des adaptations aux contraintes externes (réglementation, évolution technique, demande du client...). Le couple produit/filière est donc l'objet de démarches permanentes d'ajustement et d'optimisation.

Pour aborder la complexité d'un système, la décomposition est une approche standard [BRO 02]. Le produit et la filière étant structurés de manière hiérarchique, une décomposition est alors envisageable. Comme tout système, la filière peut être définie non seulement par sa décomposition en activités mais aussi par la manière dont celles-ci fonctionnent ensemble [BRO 02].

La filière étant composée d'une succession de processus, il existe alors une dualité produitprocessus au sein de celle-ci [EYN 97]. En effet, les données relatives au produit résultent des activités de conception et de fabrication et ces dernières exploitent les données du produit pour leur déroulement.

Selon [CAS 09], le produit interagit individuellement avec son système de production. Le produit supporte (matérialise) l'ensemble des données le concernant [KAR 03 ; MOR 03]. Ces données sont d'ordre techniques mais également opérationnelles, liées au déroulement des activités de fabrication. Ainsi, la représentation des données relatives au produit renseigne et décrit l'enchaînement des activités composant le processus et, si l'on considère plusieurs acteurs, également sa filière.

Pour résumer, le produit et les processus de la filière sont étroitement liés. La décomposition du produit permet de collecter des données, nécessaires à la conception optimale de sa filière [FIS 97] à un instant donné. Ainsi, cette dernière est adaptée au produit qu'elle supporte. Il y a alors une cohérence stratégique, appelée concordance, entre les caractéristiques du produit et les caractéristiques de la filière [GLI 16].

\subsection{Filière agile et agilité de filière}

\subsubsection{Filière agile, reflet du comportement de la filière à un instant précis}

Plusieurs recherches ont porté sur le lien produit / filière [LEE 02 ; VON 06]. Dans leurs recherches, [VON 06] mettent en évidence que la typologie de la filière évolue au cours du cycle de vie d'un produit (figure 1). Ils différencient les filières lean des filières agiles :

Une filière lean est constituée d'acteurs qui individuellement se concentrent sur la suppression des étapes à non-valeur ajoutée. Les entreprises maîtrisent les techniques de lean manufacturing et collaborent avec les autres membres de la filière pour gagner en performance [LEE 02]. Une filière lean rencontre des difficultés à s'adapter aux changements de l'environnement (norme ou demande des clients par exemple). Les alliances entre les entreprises se font sous la forme de partenariats, les changements d'acteurs au sein d'une filière lean étant peu fréquents.

Une filière agile est constituée d'acteurs qui individuellement comprennent les exigences clients [LEE 02 ; CHR 00] grâce à une interface avec le marché. Les entreprises ont aussi une connaissance 
collective qui s'exprime lors d'activités communes (colloques, rassemblement au sein de structures professionnelles type syndicat, programme de recherche au sein d'entité partagée) ou bilatérale (relation client-fournisseur au cours desquelles des informations client sont discutées).Une filière agile a la capacité à s'adapter rapidement aux futurs changements (capacité d'investissement, élaboration de normes...), les alliances entre les entreprises sont dynamiques pour fabriquer de nouveaux produits. Les partenaires peuvent fréquemment variés selon les offres et la demande client.

Notons qu'une filière peut adapter son comportement et prendre des caractéristiques de chacune afin de former une filière hybride.

\begin{tabular}{|l|l|l|l|}
\hline \multicolumn{1}{|c|}{ Product Type } & Standard & Innovative & Hybrid \\
\hline Product Life Cycle & & $\begin{array}{c}\text { Agile Supply } \\
\text { Chain }\end{array}$ & \\
\cline { 1 - 1 } Introduction & \multirow{2}{*}{$\begin{array}{c}\text { Lean Supply } \\
\text { Chain }\end{array}$} \\
\cline { 1 - 1 } Growth & $\begin{array}{c}\text { Hybrid / Lean } \\
\text { Cupply Chain }\end{array}$ & \\
\hline Maturity & Decline & & \\
\hline
\end{tabular}

Figure 1. Classification des filières basée sur le type de produit et sur le cycle de vie du produit [VON 06]

Les travaux de [FIS 97 ; LEE 02] et la classification proposée par [VON 06] suggèrent que les produits innovants doivent évoluer dans une filière agile lors de leur lancennent sur le marché (figure 1). Si cette hypothèse est exacte, l'entreprise innovante doit s'assurer que la future filière qui va supporter le nouveau produit sera agile, ce qui suppose de construire une filière avec de nouvelles caractéristiques garantissant son caractère agile. Cette construction souligne la capacité d'une entreprise à anticiper sa future filière, ou bien, à agir « chemin faisant» [MAR 17]. Si une innovation émerge dans une filière préalablement lean ou hybride, une mutation est alors nécessaire.

Au-delà du concept de filière agile, on peut considérer un autre principe associé : celui d'agilité qui concerne la capacité d'une filière à passer de l'état lean/hybride à celui d'agile. L'agilité d'une filière correspond donc à sa capacité à changer de configuration selon les plans stratégiques, technologiques, organisationnels ou humains des entreprises innovantes qui la composent. Une filière, et ses entreprises, font face à des innovations ou les engendrent, elles s'adaptent (ou non) à des marchés fluctuants et anticipent (ou non) différents aléas de l'environnement [CHR 04]. Par ailleurs, elles sont aptes à connaitre des phases où l'optimisation est la stratégie principale. Cela demande une agilité collective à l'échelle de la filière [PAC 04], qui peut amener cette dernière à se reconfigurer. L'agilité opère donc à plusieurs niveaux, au niveau de l'entreprise et au niveau de la filière, cette dernière s'exprime par l'agilité de ses parties prenantes, en amont et en aval de l'entreprise focale [CHR 04].

\subsubsection{Agilité de la filière, reflet de la nature dynamique des filières}

Les filières sont des systèmes dynamiques. Pour atteindre une concordance entre les caractéristiques du produit et les caractéristiques de la filière, [GLI 16] suggère que les entreprises ajustent rapidement leurs filières à mesure que les produits se déplacent le long de leur cycle de vie. De par son degré de nouveauté, un produit innovant occasionne des incertitudes et des changements dans son environnement. Pour les minimiser, la filière doit être capable de fournir des réponses organisationnelles efficaces et efficientes [FAY 16; PUR 14] pour satisfaire les caractéristiques de son nouveau produit.

Un fort niveau d'incertitude complique la concordance entre les caractéristiques du produit et celles de la filière. L'agilité de la filière permet de minimiser l'incertitude inhérente à l'environnement et facilite la réalisation de la concordance produit-filière sans toutefois permettre de concevoir la filière adaptée au produit [GLI 13]. 
L'agilité influence l'orientation stratégique et opérationnelle des entreprises et leurs interactions au sein d'une filière [JIN 03; FAY 16]. L'agilité de la filière souligne la capacité des entreprises à apporter des ajustements rapides à leurs filières pour répondre ou s'adapter aux changements [GLI 13], aux incertitudes internes et externes. Pour cela, elle s'appuie sur une intégration efficace des relations au sein de la filière [FAY 16] et sur une reconfiguration de leurs ressources de manière collaborative [SHA 06] pour fonctionner de manière plus efficace en fonction des caractéristiques du produit qu'elles manipulent [GLI 16]. L'agilité de la filière est une source d'avantage concurrentiel pour les entreprises de cette la filière [GLI 13] qui cherchent à coordonner leurs activités pour atteindre ensemble un niveau d'agilité supérieur à celui de leurs concurrents [LIN 06].

Pour conclure, l'agilité de la filière peut être considérée comme un paradigme de changement dans la gestion de la filière [FAY 16].

\section{Démarche de recherche}

\subsection{But de la recherche}

Cette recherche a deux objectifs :

- Montrer qu'une entreprise innovante peut reconfigurer et rendre agile sa filière pour l'adapter aux spécifications du nouveau produit et s'assurer de son succès lors de son lancement sur le marché.

- Mieux décrire les deux concepts « filière agile » et « agilité de filière » en se basant sur le cas de la Swatch.

L'objectif est de décrire les phénomènes ayant affectés le couple produit/filière. Pour les illustrer, une recherche par étude de cas a été menée. La montre Swatch, montre à quartz d'entrée de gamme, a été étudiée afin de visualiser comment la filière initiale suisse des montres à quartz haut de gamme a été transformée suite au développement de cette montre innovante.

Afin de concurrencer l'émergence des montres japonsaises sur le marché, la montre Swatch est développée au sein d'une société spécialisée dans la fabrication de mouvement. Il s'agit d'une montre en plastique dont le mécanisme a été simplifié. Son développement a conduit à un changement radical dans les activités de l'entreprise, mais également dans celles de la filière : la fabrication a changé, la distribution a évolué.

Le choix de la Swatch comme étude de cas se justifie de plusieurs manières. Premièrement, son processus de fabrication est totalement nouveau dans l'industrie horlogère. Deuxièmement, la filière initiale était organisée sous la forme d'un cartel. L'émergence de la Swatch a eu une incidence sur l'organisation de la filière, les rôles ont été redistribués.

De plus, la Swatch est un sujet de recherche qui a fait l'objet de nombreuses études pour approfondir d'autres thématiques : la littérature le concernant est donc riche, la collecte des données est accessible et vérifiable ce qui permet une étude approfondie du produit innovant et de sa filière.

Dans cette recherche, la filière est étudiée sous une forme fonctionnelle. Cela signifie que la filière est décrite comme une séquence d'activités ordonnées, conduisant à l'étude de la filière d'un point de vue «processus ». La collecte de données se concentre alors sur les choix de techniques issus du produit, les processus de fabrication ...

\subsection{Méthodologie}

\subsubsection{Collecte de données}

La collecte des données s'est portée exclusivement sur des documents scientifiques (publications et livres) à l'exclusion donc de documents de vulgarisation ou de presse grand public. Chaque donnée 
collectée a été corroborée par au moins deux documents scientifiques. Ces données sont utilisées pour décomposer le produit et ensuite modéliser la filière correspondante.

Plus précisément, le produit initial et le produit innovant sont décomposés en composants. Chaque composant (ou ensemble de composants) est défini selon ses caractéristiques, c'est-à-dire :

- La ou les matières premières.

- Le ou les composants périphériques.

- Le ou les procédés de fabrication, qui caractérise(nt) le changement d'état opéré.

- L'interfaçage avec les autres composants.

\subsubsection{Traitement des données}

Le traitement des données se fait en deux phases.

\section{Phase une : de l'architecture produit à l'identification des procédés}

Cette première phase consiste à étudier l'architecture d'un produit pour en déduire la séquence de procédés conduisant à la fabrication de ce produit. Un procédé caractérise un changement d'état physique, c'est une méthode ou une technique qui conduit à la fabrication d'un matériau ou d'un produit fini. Un procédé est donc une activité spécifique d'un processus.

La décomposition se fait du produit fini vers la matière première, en collectant les procédés de fabrication de chaque composant, groupe de composants et interfaces. Ainsi, il est possible d'établir la séquence de procédé permettant de passer de la matière première au produit fini.

\section{Phase deux : des procédés aux processus}

Dans la seconde phase, les procédés définis précédemment sont uniformisés et intégrés au sein de processus. Ces processus sont composés d'une succession d'activités et de procédés, décrivant précisément et logiquement les étapes de fabrication d'un composant ou d'un produit. Afin d'utiliser un thesaurus de termes techniques précis pour décrire les activités et en optant pour une terminologie usuelle pour faciliter la comparaison entre les différentes filières, nous avons opté pour le Répertoire Opérationnel des Métiers et des Emplois proposé par le Pôle Emploi. Ce référentiel recense l'ensemble des activités, compétences nécessaires à la réalisation d'un processus par profession. Nous avons plus particulièrement utilisé le vocabulaire relatif aux métiers suivants « Réparation - Montage en Système Horloger », "Ajustement et montage de fabrication» ou "Réglage d'équipement de formage de plastique ».

De cette manière, la séquence de processus a été établie pour déterminer la forme de la filière du futur produit.

\subsubsection{Modélisation de la filière}

Les informations collectées permettent de modéliser la filière. La méthodologie Harmony for System Engineering couplée à l'outil Rational Rhapsody ${ }^{\circledR}$ a été privilégiée car elle permet d'obtenir plusieurs diagrammes, décrivant ainsi le comportement de la filière à l'aide d'un langage SysML. Pour cette étude, uniquement les diagrammes d'activités seront présentés afin de mettre en évidence la succession de processus et les liens entre les activités de la filière.

A cette étape, deux filières sont modélisées : la filière de la montre à quartz traditionnelle et la filière de la Swatch. 
La modélisation obtenue souligne les processus inhérents à chaque filière. Ces processus peuvent être comparés deux à deux afin de visualiser ceux qui sont communs, modifiés ou différents. En analysant les stratégies mises en œuvre par l'entreprise innovante, les ajustements effectués pour passer de la filière initiale à la nouvelle filière sont également mis en évidence. La figure 2 résume la méthodologie utilisée dans notre recherche.

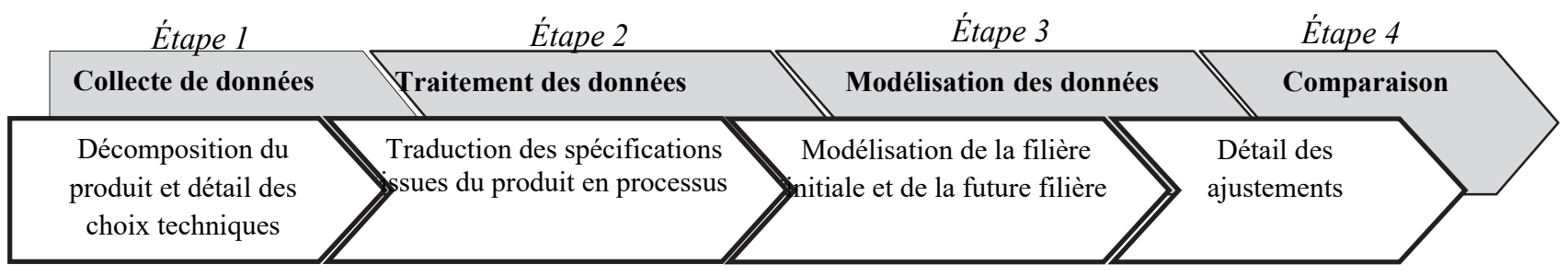

Figure 2. Méthodologie mise en œuvre pour modéliser et comparer les filières

\section{Résultats}

\subsection{Présentation du cas d'étude}

A l'origine, l'industrie horlogère est organisée sous forme de cartel où chaque entreprise est impliquée dans une seule tâche spécifique sans possibilité de combiner d'autres tâches. Seule l'expertise de l'entreprise est mise en valeur. De ce fait, une multitude d'entreprises [AGUI 04] sont nécessaires pour fabriquer une montre à quartz traditionnelle.

En investissant dans des activités de recherche et développement concernant des activités assumées par d'autres acteurs, une entreprise de mouvements a conçu la Swatch et a ainsi remis en cause l'industrie horlogère suisse.

La Swatch est une montre à quartz à aiguilles, en plastique soudée, robuste, artistique mais fonctionnelle, très peu coûteuse à fabriquer. Cette nouvelle montre comporte une cinquantaine de composants, contrairement aux montres à quartz traditionnelles qui en comptent plus d'une centaine. Cependant, elle conserve la qualité et la durabilité des montres suisses traditionnelles [GAR 12].

\subsection{Phase 1 : Décomposition du produit et détail des choix techniques}

La montre à quartz traditionnelle est réalisée en trois parties :

- Un mouvement constitué d'une platine sur laquelle sont fixés les composants horlogers : ressort, rouage, roue d'échappement, balancier, spirale...

- Un organe d'affichage (cadran et aiguilles) qui vient se fixer sur le mouvement.

- Un boîtier dans lequel viennent s'emboiter le mouvement et l'organe d'affichage. Le verre et le bracelet sont fixés mécaniquement.

La fabrication de cette montre est très complexe : les pièces se montent par le dessus, par le dessous ou sur les côtés. De ce fait, la montre ne peut être réalisée que manuellement [GAR 12].

La Swatch a une architecture totalement différente afin de pouvoir automatiser sa fabrication, elle est également réalisée en trois parties :

- Un mouvement dont le nombre de composants a diminué.

- Un organe d'affichage. 
- Un boitier en plastique injecté dont le fond sert de support pour le mouvement (en remplacement de la platine). Les composants du mouvement sont greffés sur le boîtier. Le boîtier et le bracelet ne font plus qu'un, grâce à un procédé d'injection plastique et la glace est soudée par ultrason au boîtier.

Ces choix techniques impliquent une fabrication unidirectionnelle de la montre unidirectionnelle, les composants sont montés uniquement par empilement vertical.

Les décompositions d'une montre traditionnelle et d'une montre Swatch sont résumées dans le tableau 1.

\begin{tabular}{|c|c|c|c|}
\hline $\begin{array}{l}\text { Partie de la } \\
\text { montre }\end{array}$ & Fonction & $\begin{array}{c}\text { Solution envisagée en termes de } \\
\text { composants (Montre traditionnelle) }\end{array}$ & $\begin{array}{c}\text { Solution envisagée en termes de } \\
\text { composants (Swatch) }\end{array}$ \\
\hline \multirow{4}{*}{ Mouvement } & Accumulateur d'énergie & Ressort & Ressort \\
\hline & $\begin{array}{c}\text { Organes de transmission } \\
\text { (Ébauche) }\end{array}$ & Rouage, platine & Rouage \\
\hline & Organe de distribution & Roue d'échappement & Roue d'échappement \\
\hline & Organe régulateur & Balancier, spiral & Balancier, spiral \\
\hline Affichage & Organe d'affichage & Cadran, aiguille & Cadran, aiguille \\
\hline Habillage & Protection de l'ensemble & $\begin{array}{c}\text { Boîtier métallique, Glace, Bracelet } \\
\text { métallique }\end{array}$ & Boîtier plastique, Glace \\
\hline
\end{tabular}

Tableau 1. Décomposition de la montre à quartz traditionnelle et de la Swatch

La figure 3 compare l'assemblage d'une montre à quartz traditionnelle et d'une Swatch [MUL 83] : 


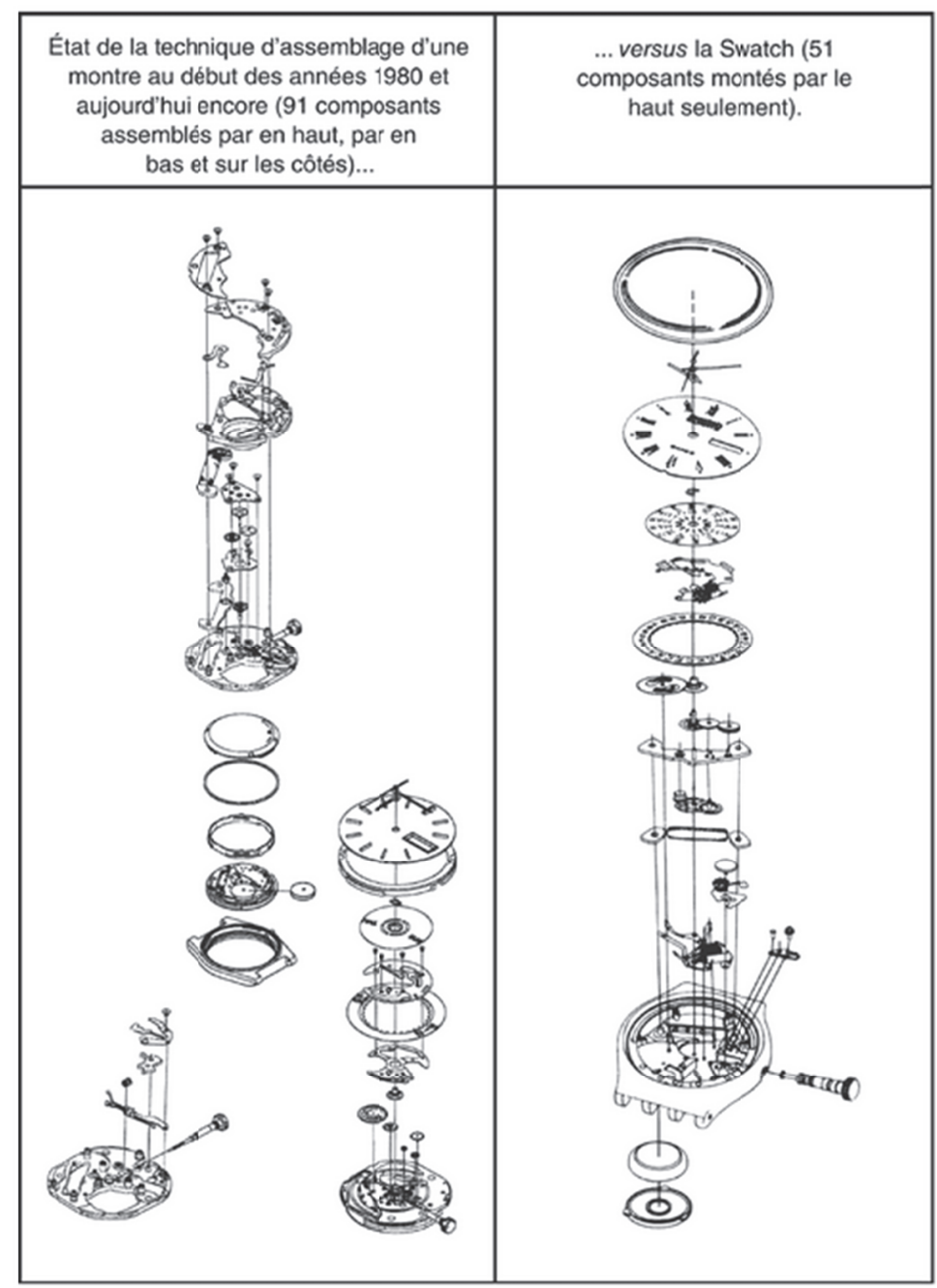

Figure 3. Comparaison de l'assemblage d'une montre à quartz traditionnelle et de la montre Swatch [MUL 83]

\subsection{Phase 2 : Traduction des spécifications issues du produit en processus}

La méthode utilisée pour traduire les spécifications issues du produit en processus est identique pour les deux produits étudiés. Afin d'éviter les redondances, seul le cas de la Siwatch sera illustré ici. Le tableau 2 synthétise les choix techniques privilégiés pour chaque composant ainsi que leur traduction en processus.

En étudiant l'architecture du produit, trois modules apparaissent : les composants participant au mouvement, ceux à l'affichage et ceux destinés à l'habillage. L'assemblage de ces trois modules forme la montre Swatch. La bibliographie a permis de lister 15 procédés principaux mis en œuvre pour concevoir et assembler les composants. Les informations obtenues ont conduit à identifier 20 processus nécessaires à la fabrication de la montre Swatch. Ces processus, qui seront détaillés en activités lors de la modélisation, forment la filière du produit. 


\begin{tabular}{|c|c|c|}
\hline $\begin{array}{l}\text { Architecture du produit } \\
\text { (simplifiée) }\end{array}$ & Procédés de fabrication & Processus \\
\hline Ressort & \multirow{5}{*}{$\begin{array}{l}\text { Usinage micro-mécanique } \\
\text { Finitions, polissage, microbillage }\end{array}$} & \multirow{5}{*}{$\begin{array}{l}\text { Achat de matière première - } \\
\text { alliage de métaux } \\
\text { Opération de micro-mécaniques }\end{array}$} \\
\hline Rouage & & \\
\hline Roue d'échappement & & \\
\hline Balancier & & \\
\hline Spirale & & \\
\hline Mouvement & Assemblage de pièces d'horlogerie & Montage en système horloger \\
\hline Cadran & $\begin{array}{l}\text { Découpe dans une plaque de laiton } \\
\text { Perçage des trous, polissage et mise en } \\
\text { couleur par galvanoplastie }\end{array}$ & \multirow{2}{*}{$\begin{array}{l}\text { Achat de matière première - } \\
\text { plaque de laiton } \\
\text { Opération de micro-mécanique } \\
\text { Ajustement et montage de } \\
\text { fabrication } \\
\text { Conduite de traitement par } \\
\text { dépôt de surface }\end{array}$} \\
\hline Aiguille & $\begin{array}{l}\text { Découpe dans une bande de laiton } \\
\text { Usinage par diamantage } \\
\text { Rivetage des aiguilles sur un tube ne } \\
\text { laiton } \\
\text { Traitement galvanique }\end{array}$ & \\
\hline Système horloger & Assemblage du cadran et des aiguilles & $\begin{array}{l}\text { Opération d'assemblage du } \\
\text { mouvement, du cadran et des } \\
\text { aiguilles } \\
\text { Contrôle de qualité }\end{array}$ \\
\hline Glace & $\begin{array}{l}\text { Traitement anti-reflet } \\
\text { Biseautage des angles }\end{array}$ & $\begin{array}{c}\text { Achat de matière première - } \\
\text { verre } \\
\text { Réalisation d'objets } \\
\text { fonctionnels en verre }\end{array}$ \\
\hline Boitier & Injection plastique sous pression & $\begin{array}{l}\text { Achat de matière première - } \\
\text { poudre plastique / pigments } \\
\text { Formage des plastiques } \\
\text { Expertise couleur en industrie }\end{array}$ \\
\hline Montre Swatch & $\begin{array}{l}\text { Fixation du système horloger dans le } \\
\text { boîtier } \\
\text { Soudage de la glace par ultrason }\end{array}$ & $\begin{array}{l}\text { Assemblage mécanique } \\
\text { Soudure par ultrason } \\
\text { Contrôle qualité } \\
\text { Conditionnement } \\
\text { Vente en gros } \\
\text { Vente au détail }\end{array}$ \\
\hline
\end{tabular}

Tableau 2. Traduction des spécifications issues du produit en processus

\subsection{Phase 3 : Interprétation des modèles de filière}

Le traitement des données a permis de transformer les spécifications du produit en caractéristiques de la filière. La modélisation de la filière sous forme de flux fonctionnel est alors réalisable. 
La filière va être étudiée selon trois angles : l'approvisionnement des matières premières et des composants, la fabrication du produit et la vente de ce produit. Seule la modélisation des processus de fabrication de chacune des montres sera présentée dans cet article. Chacun de ces processus est décomposé en activités.

\subsubsection{Au niveau de l'approvisionnement}

Le changement de matière première pour le boitier de la montre impacte l'approvisionnement au niveau des acteurs. Les fournisseurs de matière métallique (boitier et système d'attache) disparaissent au profit de fournisseurs de poudre plastique. Les processus d'approvisionnement en alliage de métaux (composants horlogers), laiton (cadran et aiguilles) et verre restent identiques, en termes d'activités et d'acteurs.

\subsubsection{Au niveau de la fabrication}

Les processus de fabrication du cadran et des aiguilles ainsi que celle de la glace sont restés identiques.

Dans un souci de réduction des coûts et de maitrise de la qualité, la fabrication de certains composants (la platine par exemple) n'est plus nécessaire ce qui permet de diminuer le nombre d'activités en parallèle sans pour autant supprimer le processus.

Par contre, le processus de fabrication du boîtier est complètement nouveau, remplaçant à la fois les processus de fabrication du boîtier métallique et du bracelet métallique. L'automatisation du processus de fabrication du boîtier diminue le nombre d'activités à réaliser. De ce fait les fournisseurs d'équipements et les sociétés de service (maintenance entre autres) sont changés.

L'assemblage de la montre est également impacté. Dans les montres à quartz traditionnelles, les composants horlogers sont assemblés dans plusieurs directions puis emboîtés dans le boitier. Dans la montre Swatch, les composants horlogers sont assemblés de manière unidirectionnelle et l'activité d'emboitage est supprimée. La fabrication et l'assemblage sont automatisés et industrialisés. Des contrôles qualité sont intégrés à chaque étape pour vérifier le bon fonctionnement de l'assemblage effectué. De même que pour le boitier, les évolutions de l'assemblage s'accompagnent de changements de fournisseurs d'équipements et de sociétés de service (maintenance entre autres).

La tableau 3 illustre les changements opérés au sein du processus d'assemblage de la montre. 


\section{Légende}

Le processus et les acteurs impliqués dans ce processus sont identiques

Le processus initial a été modifié (apparition de nouvelles activités)

Le processus est nouveau dans la filière finale

Le processus est supprimé dans la filière initiale

\section{Montre à quartz traditionnelle}

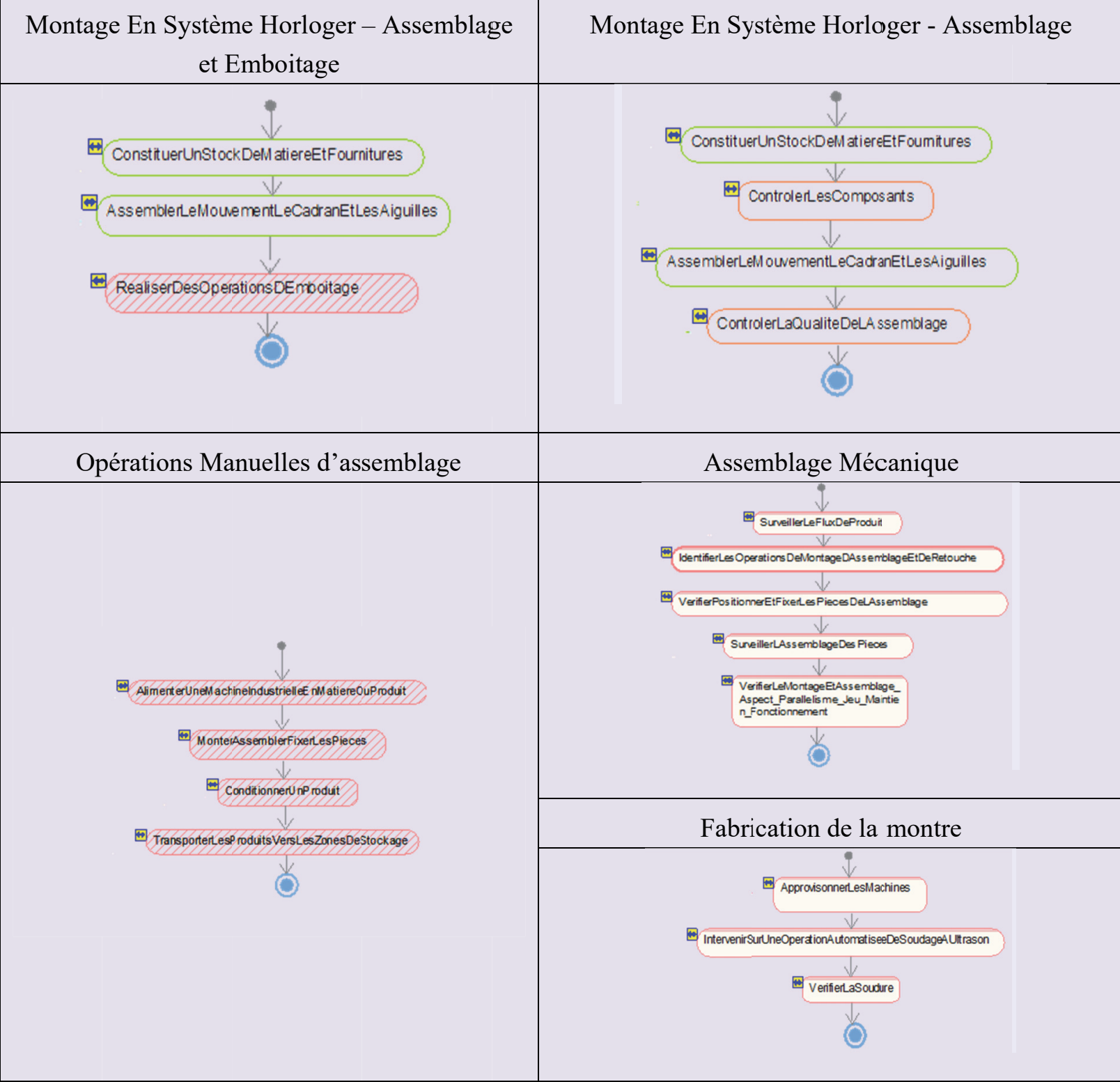

Tableau 3. Transformation au sein du processus d'assemblage de la montre

\subsubsection{Au niveau de la vente}

La soudure opérée en fin d'assemblage rend la montre irréparable, supprimant ainsi le processus de service après-vente dans la filière de la Swatch. De nombreux distributeurs, à l'instar du réseau de Tissot, ainsi que des détaillants horlogers suisses se sont montrés réticents à l'idée de vendre des montres non réparables. L'entreprise a donc dû trouver d'autres distributeurs comme des grandes surfaces ou des magasins de sport. Le processus de vente est resté le même, mais de nouveaux acteurs en aval se sont intégrés à la filière. 


\subsection{Phase 4 : Détail des ajustements}

Pour ajuster la filière initiale, de nombreuses décisions ont mises en œuvre au sein de la filière par les managers de l'entreprise innovante. Le tableau 4 met en évidence les décisions mises en œuvre pour organiser la filière la plus optimale.

Besoin de la nouvelle filière

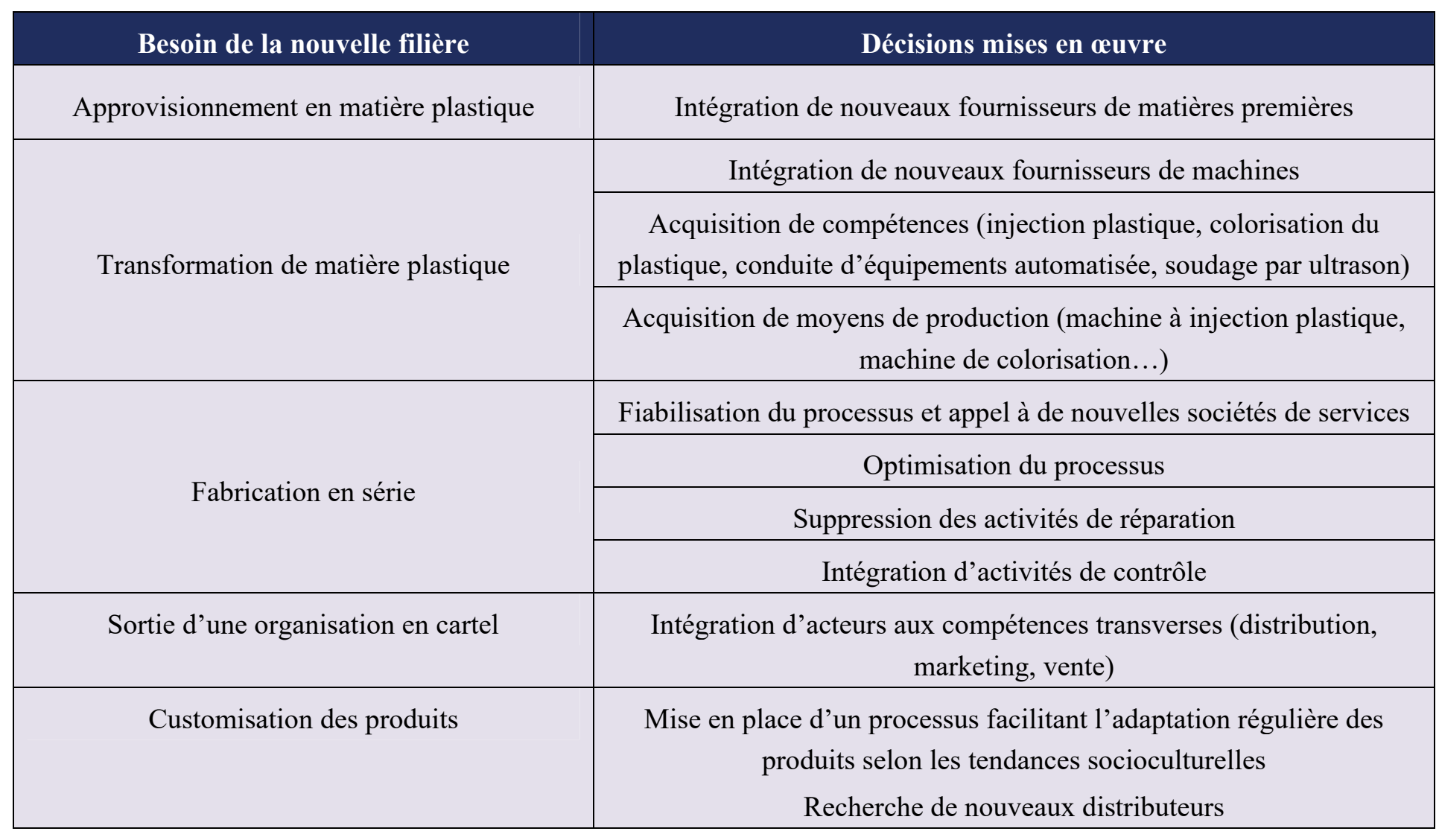

\section{Tableau 4. Décisions mises en œuvre dans l'organisation de la future filière}

Le choix de l'injection plastique nécessite de nouveaux besoins en termes d'approvisionnement en matière première. De nouveaux fournisseurs, spécialisés dans la poudre plastique, ont intégré la filière de la Swatch remplaçant ainsi les fournisseurs en matières métalliques : ils ont été approchés et mis en concurrence par les concepteurs de la Swatch.

Le nouveau procédé de fabrication conduit à des décisions d'investissement dans des machines spécialisées : des machines à injection, des machines à souder à ultrason... Ainsi des partenariats ont été noués avec des fabricants d'équipements (moules, automatisme en particulier) pour créer les installations nécessaires. De plus, de nouvelles compétences sont nécessaires pour faire fonctionner ces installations industrielles, elles ont été acquises par formation ou par embauche.

La soudure verre-boîtier a permis de renforcer la résistance mécanique de la montre mais également de fiabiliser le processus de fabrication et permettre une production en série. En effet, la soudure empêche toute réparation, demandant ainsi une grande maîtrise de la fabrication afin de fabriquer sans défaut. Cela n'était possible qu'avec une architecture simplifiée de la montre, conduisant ainsi à une diminution du coût de revient de la montre [GAR 12]. Cette fabrication zéro-défaut a fait disparaitre les acteurs extérieurs spécialisés en réparation.

L'objectif de la Swatch n'est pas de concurrencer les montres à quartz suisse traditionnelle [GAR 12]. De ce fait, la Swatch n'a pas remplacé la montre à quartz traditionnelle, la filière initiale est donc toujours d'actualité. Cependant, l'émergence de la Swatch a redistribué les rôles au sein de la filière des montres à quartz traditionnelles. Notons que la vision « processus » privilégiée ici ne permet pas de 
montrer comment les acteurs de la filière initiale se sont réorganisés autour des processus pour fabriquer les montres d'origine.

Pour mettre fin à l'organisation en cartel, des compétences ont été intégrées à la nouvelle filière sous forme d'embauche, de sollicitations d'acteurs externes (designer et spécialiste en marketing) ou de partenariats (distributeurs et revendeurs). Certains de ces acteurs n'étant pas des spécialistes de l'horlogerie mais des acteurs ayant des compétences transverses.

La Swatch étant un accessoire de mode, elle s'adapte aux différences tendances de l'environnement. Son aspect évolue souvent au rythme des saisons et des évènements. Le processus « Expertise Technique Couleur » permet aisément de changer l'aspect visuel (en termes de couleur) des différents produits. Ce processus, maîtrisé et réalisé par l'entreprise innovante, souligne la volonté de l'entreprise de rester en contact avec le marché.

\section{Discussion}

La modélisation permet de mieux appréhender la manière dont s'organise la filière d'un produit et synthétise à un instant $t$ les résultats de l'agilité de la filière. Anticiper la conception d'une filière nécessite une réflexion sur les décisions dans les phases amont de l'innovation. Le cas de la Swatch en est un parfait exemple, les choix sur le produit (procédés, types de production) ont impacté la configuration de la future filière.

\section{1. État de la filière initiale}

La modélisation de la filière de la montre à quartz traditionnelle a montré que cette dernière faisait appel à de nombreux processus différents, à de nombreux endroits différents (conséquence de la cartellisation). Au moment de l'émergence de la Swatch, la filière de la montre à quartz traditionnelle est de type hybride : la séquence de processus est bien maîtrisée par l'ensemble de la filière conduisant à un renouvellement des acteurs presque inexistant mais l'assemblage à la main autorise une personnalisation du produit.

Cependant, cette filière n'est pas adaptée à la Swatch, de par son organisation mais également et majoritairement de par sa séquence de processus. La filière de la montre Swatch doit alors se construire pour permettre la fabrication de ce nouveau produit. Les actions mises en œuvre dans le cadre de sa construction ont conduit à la fin de la cartellisation de l'industrie horlogère. La filière des montres à quartz traditionnelles reste cependant inchangée en termes de processus.

\subsection{Agilité de la filière}

La Swatch, via ses caractéristiques et son procédé de fabrication, est une innovation. De ce fait, des incertitudes apparaissent, notamment concernant son acceptation sur le marché, et le procédé de fabrication privilégié perturbe la filière en place. S'orienter vers la mise en place d'une filière agile est une décision stratégique cohérente pour minimiser les impacts des incertitudes du marché et les perturbations occasionnées par la Swatch [SHA 17 ; VON 06]. Pour y parvenir, les entreprises de la filière ont mis en place des actions spécifiques pour rendre la future filière agile et permettre une concordance entre la Swatch et sa filière.

De ce choix stratégique découle des décisions d'ordre stratégiques et opérationnelles.

En précisant le type de filière visée, les décisions stratégiques soulignent l'orientation que prend la filière, son ouverture sur le marché, son mode de fonctionnement. Elles renseignent sur les relations entre les acteurs et sont étroitement liées à la gouvernance de la filière. Ces décisions engagent les entreprises sur le long terme et attestent de l'agilité de la filière initiale. 
Les décisions stratégiques ont eu un impact direct sur les acteurs de la filière. Le choix de concevoir une montre en plastique a permis d'intégrer de nouveaux fournisseurs : des fournisseurs plasturgiques, des équipementiers de machine à injection et soudage plastique ainsi que des machines de colorisation. La Swatch étant un produit d'entrée de gamme, l'intégration de nouveaux distributeurs permet d'élargir les points de vente et ainsi de la rendre accessible au plus grand nombre. En rendant la Swatch irréparable, l'entreprise a délibérément supprimé les activités de réparation.

Les décisions opérationnelles concernent les processus, les acteurs, les compétences et les équipements. Leur recensement est essentiel pour modéliser la filière, pour comprendre le cheminement des tâches qui mènent à la réalisation d'un produit. Ces décisions engagent les entreprises de la filière à court terme.

Les décisions opérationnelles pour mettre en place la future filière découlent des choix techniques privilégiés pour la fabrication de la Swatch. Les choix du procédé d'injection plastique et du soudage par ultrason ont une incidence directe sur la mise en place des processus (acteurs concernés, compétences et équipements nécessaires).

Le tableau 5 résume les caractéristiques de chacune des décisions :

\begin{tabular}{|c|c|c|}
\hline & Décisions stratégiques & Décisions opérationnelles \\
\hline Objectifs & $\begin{array}{l}\text { Définition de l'orientation de la filière, } \\
\text { de son ouverture sur le marché et de son } \\
\text { mode de fonctionnement }\end{array}$ & $\begin{array}{l}\text { Gestion des processus, des liens } \\
\text { client/fournisseur dans la filière, } \\
\text { des partenariats, des compétences } \\
\text { et des équipements }\end{array}$ \\
\hline But & $\begin{array}{l}\text { Organiser la gouvernance de la filière : } \\
\text { gestion des relations entre les différents } \\
\text { acteurs }\end{array}$ & $\begin{array}{l}\text { Mise en place des tâches } \\
\text { essentielles à la réalisation du } \\
\text { produit acteur par acteur ou par } \\
\text { duo d'acteurs }\end{array}$ \\
\hline Impact & Filière globale & Quelques acteurs \\
\hline Fréquence & Ponctuelle voire unique & Fréquentes et prévisibles \\
\hline Vision prospective & Long terme & Court terme \\
\hline $\begin{array}{c}\text { Nature de la } \\
\text { décision }\end{array}$ & Incertaine & Précise \\
\hline
\end{tabular}

Tableau 5. Caractéristiques des décisions stratégiques et opérationnelles

Les décisions prises au niveau de la filière participent aux succès de la Swatch. Les décisions stratégiques, de par leur unicité, ont permis d'organiser et de modeler la filière tandis que les décisions opérationnelles, de par leur fréquence, font fonctionner la filière. Les décisions opérationnelles supportent les décisions stratégiques, il s'agit d'une réponse opérationnelle à la stratégie décidée par l'entreprise. Elles ont deux rôles :

- Elles correspondent aux actions mises en œuvre pour concevoir la filière de la Swatch.

-Elles favorisent l'agilité de la filière.

Le tableau 6 montre le type de décisions privilégiées pour mettre en place et faire fonctionner la filière de la Swatch. 


\begin{tabular}{|c|c|c|}
\hline $\begin{array}{l}\text { Approvisionnement en matière } \\
\text { plastique }\end{array}$ & $\begin{array}{l}\text { Intégration de nouveaux fournisseurs de matières } \\
\text { premières }\end{array}$ & Stratégique \\
\hline \multirow{3}{*}{$\begin{array}{l}\text { Transformation de matière } \\
\text { plastique }\end{array}$} & Intégration de nouveaux fournisseurs de machines & Stratégique \\
\hline & $\begin{array}{l}\text { Acquisition de compétences (injection plastique, } \\
\text { colorisation du plastique, conduite d'équipements } \\
\text { automatisée, soudage par ultrason) }\end{array}$ & Opérationnelle \\
\hline & $\begin{array}{l}\text { Acquisition de moyens de production (machine à } \\
\text { injection plastique, machine de colorisation...) }\end{array}$ & Opérationnelle \\
\hline \multirow{4}{*}{ Fabrication en série } & Fiabilisation du processus & Opérationnelle \\
\hline & Optimisation du processus & Opérationnelle \\
\hline & Suppression des activités de réparation & Stratégique \\
\hline & Intégration d'activités de contrôle & Opérationnelle \\
\hline Sortie d'une organisation en cartel & $\begin{array}{l}\text { Intégration d'acteurs aux compétences transverses } \\
\text { (distribution, marketing, vente) }\end{array}$ & Stratégique \\
\hline Customisation des produits & $\begin{array}{c}\text { Mise en place d'un processus facilitant l'adaptation } \\
\text { régulière des produits selon les tendances } \\
\text { socioculturelles }\end{array}$ & $\begin{array}{l}\text { Stratégique / } \\
\text { Opérationnelle }\end{array}$ \\
\hline
\end{tabular}

Tableau 6. Type des décisions mises en œuvre dans l'organisation de la future filière

L'intégration de nouveaux fournisseurs et d'acteurs transverses, la suppression des activités de réparations et le choix de s'adapter aux tendances socioculturelles sont des décisions stratégiques. L'acquisition de compétences et de moyens de production, les actions portant sur le processus de fabrication (fiabilisation, optimisation et contrôle) sont des décisions opérationnelles qui font fonctionner la filière.

Ces différentes décisions ont permis à la filière de développer ses capacités pour passer d'une forme hybride à agile, principalement l'intégration d'acteurs aux compétences transverses ainsi que la mise en place du processus de customisation des produits. Cette nouvelle filière a la capacité de :

- comprendre les exigences clients grâce à une interface avec le marché (collaboration étroite avec des designers externes spécialistes des tendances de modes, processus de customisation),

- s'adapter et s'ajuster rapidement en se basant sur les habitudes des consommateurs suisses. En effet, lors du lancement de la Swatch sur le marché suisse, le retour des clients a permis de fiabiliser progressivement le processus de fabrication, les montres étant sous garantie. La configuration de la filière de la Swatch s'est améliorée petit à petit.

Notons que la Swatch a été rapidement plébiscitée sur le marché suisse, le caractère agile atteint semble avoir suffi dans ce cas de figure.

La filière obtenue supporte : un produit industriel compétitif et Swiss Made avec un fonctionnement identique aux montres à quartz traditionnelles dont le coût de fabrication est minime [GAR 12]. Le tableau 7 démontre que les principales orientations de cette nouvelle montre sont satisfaites par les décisions qui ont permis de concevoir la filière. 


\begin{tabular}{|c|c|}
\hline $\begin{array}{l}\text { Grandes orientations de conception pour le } \\
\text { produit }\end{array}$ & Décisions de conception de la filière \\
\hline \multirow{3}{*}{ Prix de fabrication bas } & $\begin{array}{c}\text { Intégration de nouveaux fournisseurs de matières } \\
\text { premières }\end{array}$ \\
\hline & $\begin{array}{l}\text { Intégration de nouveaux fournisseurs de } \\
\text { machines }\end{array}$ \\
\hline & Fiabilisation du processus \\
\hline \multirow[b]{3}{*}{ Montre industrielle et compétitive } & Fiabilisation du processus \\
\hline & Optimisation du processus \\
\hline & $\begin{array}{l}\text { Mise en place d'un processus facilitant } \\
\text { l'adaptation régulière des produits selon les } \\
\text { tendances socioculturelles }\end{array}$ \\
\hline \multirow{4}{*}{ Fabriquée en Suisse } & $\begin{array}{c}\text { Acquisition de compétences (injection plastique, } \\
\text { colorisation du plastique, conduite d'équipements } \\
\text { automatisée, soudage par ultrason) }\end{array}$ \\
\hline & $\begin{array}{l}\text { Acquisition de moyens de production (machine à } \\
\text { injection plastique, machine de colorisation...) }\end{array}$ \\
\hline & $\begin{array}{l}\text { Intégration d'acteurs aux compétences } \\
\text { transverses (distribution marketing, vente) }\end{array}$ \\
\hline & Développement de partenariats \\
\hline \multirow[t]{2}{*}{$\begin{array}{l}\text { Même fonctionnement qu'une montre à quartz } \\
\text { traditionnelle }\end{array}$} & $\begin{array}{c}\text { Acquisition de compétences (injection plastique, } \\
\text { colorisation du plastique, conduite d'équipements } \\
\text { automatisée, soudage par ultrason) }\end{array}$ \\
\hline & Intégration d'activités de contrôle \\
\hline
\end{tabular}

Tableau 7. Concordance entre les orientations du produit et les décisions de la filière

Le processus de fabrication a été fiabilisé au rythme des retours clients. Tout le processus est automatisé et chaque assemblage est suivi d'une opération de contrôle [GAR 12], facilitant ainsi la mise en place d'une politique de lean production. La maitrise du processus de fabrication de la Swatch a conduit à l'optimisation de la filière : une fois le produit mature, la filière se transforme pour suivre une tendance plus orientée «lean», visant ainsi à réduire les étapes à non-valeur ajoutée. Ainsi, la filière perd en agilité.

Aujourd'hui, ces différents faits soulignent l'hybridation de la filière : la mise en place de pratiques de lean manufacturing et une interface sur le marché conduisant à une différenciation des produits [VON 06].

\section{Conclusion}

Par une approche historique, il est possible de décrire les liens entre un produit et sa filière et également de visualiser les évolutions de cette dernière si le produit gagne en nouveauté. L'ambition est alors de tester l'utilisation d'outils de modélisation de filière dans les étapes amont de projets innovants et d'étudier leurs rôles en tant qu'outils d'aide à la décision : quelles transformations à opérer sur la filière d'origine pour qu'elle s'adapte au nouveau produit sont envisageables. Cette 
modélisation sert ainsi de support pour visualiser où l'agilité doit se manifester pour transformer une filière et la rendre apte à fabriquer un produit innovant.

La modélisation permet de faire un état des lieux des filières à un instant précis. Dans notre cas, il est possible d'observer la filière initiale, celle de la montre à quartz traditionnelle puis la filière de la Swatch, à son lancement. Elle permet de caractériser son caractère agile, lean ou hybride. Cependant, cette représentation reste statique. L'aspect dynamique des filières est alors représenté par l'agilité mise en œuvre pour passer d'une filière à l'autre. L'aspect agile caractérise la filière à un instant donné tandis que l'agilité de la filière souligne la capacité à mettre en œuvre des transformations pour supporter l'émergence d'une innovation et les incertitudes, perturbations associées.

Le cas de la Swatch sert de support pour comprendre comment la conception du produit impacte la conception de la filière ainsi que la manière dont l'agilité apparait comme une dynamique pour conduire une reconfiguration de filière réussie suite à une innovation. Il valide qu'une entreprise peut reconfigurer sa filière et la rendre plus agile afin de supporter la fabrication du produit innovant. De plus, ce cas met en évidence les concepts de filière agile et d'agilité de filière. Cependant, il reste limité pour bien comprendre la manière dont les décisions opèrent dans cette transformation de filière. De nombreux cas doivent être étudiés afin de valider si les éléments recensés ici sont génériques et valables pour toute autre innovation.

Plusieurs perspectives sont envisageables, notamment concernant la modélisation qui est améliorable sur différents points. La modélisation actuelle est proposée uniquement d'un point de vue processus. A partir de là, il est possible de réfléchir en termes de compétences et d'équipements, en termes d'acteurs... Les acteurs renseignés actuellement sont caractérisés uniquement selon leur typologie. Une étude approfondie des compétences et des équipements, ajoutée à l'étude des processus proposés dans cet article, peut conduire à une description plus précise encore des acteurs. De plus, la valeur créée par les activités de la filière ne sont pas mises en évidence dans cette modélisation, il est donc impossible d'estimer la valeur ajoutée globale de la nouvelle filière. Cet aspect peut être rattaché à la notion de performance proposée dans les travaux de [SHA 17] qui considèrent que l'agilité de la filière conduit à une meilleure performance. Cette étude permettrait de juger si une filière est optimale ou en progression en se basant sur des indicateurs précis. Enfin notons que les limites spatiales de la modélisation, c'est-à-dire, le nombre de niveaux d'interrelations considérés autour de l'entreprise étudiée restent à préciser.

\section{Bibliographie}

[AGU 04] AGUillaume, C., « Les horlogers suisses face à la mondialisation (1968-1983). » Cahiers de RECITS, 57-76, 2004

[BID 13] BIDET-MAYER, T AND TOUBAL, L., « A quoi servent les filières ? ». La Fabrique de l'industrie, 2013

[BON 06] BONJOUR, ERIC, AND MARYvONNE DUlmet., « Pilotage des activités de conception par l'Ingénierie Système (IS). » dans Ingénierie de la conception et cycle de vie des produits, HERMES Science, 85-105, 2006.

[BRO 02] BROWNING, T.R., AND EPPINGER, S.D., « Modeling impacts of process architecture on cost and schedule risk in product development», IEEE transactions on engineering management 49.4, 428-442, 2002.

[CAS 09] Castro, E., Mayer F., AND Pascal Lhoste P., "Contribution to Innovation Engineering: modelling the innovation process» 8ème CIGI Congrès International de Génie Industriel. 2009.

[CHR 00] CHRISTOPHER, M. « The agile supply chain: competing in volatile markets. » Industrial marketing management 29.1: 37-44, 2000.

[CHR 04] CHRISTOPHER, M., AND PECK, H., « Building the resilient supply chain.» The international journal of logistics management, 15(2), 1-14, 2004.

[CHR 92] CHRISTOPHER, M., Logistics and supply chain management, Pitman Publishing. London, UK, 1992.

[DER 75] De Rosnay, J. Le macroscope, Vers une vision globale, Edition du Seuil, 1975. 
[EYN 97] EYNARD, B., GiRARD, P., AND CHEN, D., "Un modèle produit support à la conduite de processus de conception. » Deuxième Congrès Internationale Franco-Québécois de Génie Industriel, 1997.

[FAY 16] FAYEZI, S., ZUTSHI, A., AND O'LOUGHLIN, A. « Understanding and development of supply chain agility and flexibility: A structured literature review. » International Journal of Management Reviews, 19(4), 379-407, 2017.

[FIS 97] FISHER M.L., « What is the right supply chain for your product? A simple framework can help you figure out the answer. » Harvard Business Review, 3, 105-116, 1997.

[FIX 05] FIXSON, S. K., " Product architecture assessment: a tool to link product, process, and supply chain design decisions. » Journal of operations management, 23(3), 345-369, 2005.

[GAR 02] GARCIA, R., AND CALANTONE, R., «A critical look at technological innovation typology and innovativeness terminology: a literature review. » Journal of product innovation management, 19(2), 110-132, 2002.

[GAR 12] GAREL, G, AND Mock E., La fabrique de l'innovation. Dunod. Paris, 2012.

[GER 05] Gereffi, G, Humphrey J, AND StURgeon T., «The governance of global value chains ». Review of International Political Economy 12:1:78-104, 2005.

[GLI 16] GligOR, D.M., «The Role of Supply Chain Agility in Achieving Supply Chain Fit». Decision Sciences 47 (3):524-53, 2016.

[GLI 13] Gligor D.M., Holcomb M.C, And Stank, T.P., «A Multidisciplinary Approach to Supply Chain Agility: Conceptualization and Scale Development ». Journal of Business Logistics 34 (2):94-108, 2013.

[HEN 90] HeNDERSON R.M. AND ClaRK, K.B. «Architectural Innovation: The Reconfiguration of Existing Product Technologies and the Failure of Established Firms ». Administrative Science Quarterly, 35 (1):9-30, 1990.

[JIN 03] JIN HAi L., ANDERSON A.R., AND HARRISON R.T., « The evolution of agile manufacturing ». Business Process Management Journal 9 (2):170-89, 2003.

[KAR 03] KÄRKKÄINEN M., JAN HOLMSTRÖM J., KARY FRÄMLING K., AND ARTTO K., « Intelligent products - a step towards a more effective project delivery chain ». Computers in Industry, Advanced Web Technologies for Industrial Applications, 50 (2):141-51, 2003.

[LAB 85] LABONNE, M. «Sur le concept de filière en économie agroalimentaire ». Institut Nationale de Recherche Agronomique. Montpellier: Laboratoire d'Economie et Sociologie Rurales, 1985.

[LAP 00] LAPIDE, L. « What about measuring supply chain performance » Achieving Supply Chain Excellence Through Technology, 2 (2):287-97, 2000.

[LEE 02] LEE. «Aligning Supply Chain Strategies with Product Uncertainties ». California Management Review 44 (3):105-19, 2002.

[LIN 06] LIN C-T., CHIU H., AND CHU P-Y. « Agility index in the supply chain ». International Journal of Production Economics 100 (2):285-99, 2006.

[LOR 03] LORINO, P. Méthodes et pratiques de la performance : le pilotage par les processus et les compétences. Ed. d'organisation, 2003.

[MAR 17] Marche B., Boly V., Morel L., CAMargo M., AND ORTT J.R., " Overview of phenomena occurring in supply chains during the emergence of innovation ». Supply Chain Forum: An International Journal 18 (3):150-65, 2017.

[MEI 02] MeINADIER J-P. Ingénierie et intégration des systèmes. Paris: Hermes Science Publications, 2002.

[MOR 03] Morel G., PANetTo H., ZAREMBa M., AND MAYer F., « Manufacturing Enterprise Control and Management System Engineering: paradigms and open issues ». Annual Reviews in Control 27 (2):199-209, 2003.

[MOR 77] MORIN E., La méthode: Tome 1, La nature de la nature. Paris: Points, 1977.

[MUL 83] MÜLLER J., AND. MOCK E., « Eine Revolution in der Uhrentechnik ». Neuen Zurcher Zeitung 2, 1983.

[OCO 13] O’CONNOR G.C., AND RiCE M.P., «New Market Creation for Breakthrough Innovations: Enabling and Constraining Mechanisms », Journal of Product Innovation Management 30.2: 209-227, 2013.

[PAC 04] PACHE G. «Le pilotage des chaînes logistiques multi-acteurs: une lecture critique des pratiques collaboratives ». Economies et sociétés 38 (12):2133-54, 2004.

[PUR 14] PURVIS L., Gosling J., AND NAIM M.M., « The development of a lean, agile and leagile supply network taxonomy based on differing types of flexibility ». International Journal of Production Economics 151 (Supplement C):100-111, 2014.

(c) 2018 ISTE OpenScience - Published by ISTE Ltd. London, UK - openscience.fr 
[SHA 06] SHARIFI H, ISMAIL H.S. AND I. REID I., " Achieving agility in supply chain through simultaneous "design of" and "design for" supply chain ». Journal of Manufacturing Technology Management 17 (8):1078-98, 2006.

[SHA 17] Sharma N., SAHAY B.S., Shankar R. AND SARMA P.R.S., « Supply chain agility: review, classification and synthesis ». International Journal of Logistics Research and Applications 20 (6):532-59, 2017.

[SlA 16] Slack N, Brandon-Jones A., And Johnston R., Operations Management. $8^{\text {th }}$ Edition. London: Peason, 2016.

[ULR 95] ULRICH K. " The role of product architecture in the manufacturing firm ». Research Policy 24 (3):419-40, 1995

[VON 06] VonderembSe M.A., UpPal M., HuAng S.H., AND DiSMUKeS J.P., « Designing supply chains: Towards theory development ». International Journal of Production Economics 100 (2):223-38, 2006. 